\title{
CER1 is a common target of WNT and NODAL signaling pathways in human embryonic stem cells
}

\author{
MASUKO KATOH ${ }^{1}$ and MASARU KATOH ${ }^{2}$ \\ ${ }^{1}$ M\&M Medical BioInformatics, Hongo 113-0033; ${ }^{2}$ Genetics and Cell Biology Section, \\ National Cancer Center Research Institute, Tokyo 104-0045, Japan \\ Received January 3, 2006; Accepted February 7, 2006
}

\begin{abstract}
Nodal and BMP signaling pathways network with WNT signaling pathway during embryogenesis and carcinogenesis. CER1 (Cerberus 1) and GREM3 (CKTSF1B3 or CER2) inhibit NODAL signaling through ACVR1B (ALK4) or ACVR1C (ALK7) to SMAD2 or SMAD3. GREM1 (CKTSF1B1) inhibits BMP signaling through BMPR1A (ALK3), BMPR1B (ALK6) or ACVR1 (ALK2) to SMAD1, SMAD5 or SMAD8. CER1, GREM1 and GREM3 are DAN domain (DAND) family members; however, transcriptional regulation of $D A N D$ family members by canonical WNT signaling pathway remains unclear. We searched for the TCF/LEF-binding site within the promoter region of DAND family genes, including CER1, GREM1, GREM2, GREM3 and NBL1. Because triple TCF/LEF-binding sites were identified within human CERl promoter by using bioinformatics and human intelligence, comparative genomics analyses on CERI orthologs were further performed. Chimpanzee CER1 gene, encoding 267-amino-acid protein, was identified within NW_111298.1 genome sequence. XM_528542.1 was not a correct coding sequence for chimpanzee CER1. Primate CER1 orthologs were significantly divergent from rodent Cer1 orthologs. Three TCF/LEF-binding sites within human CERI promoter were conserved in chimpanzee CERI promoter, two in cow and dog Cerl promoters, but not in rodent Cer 1 promoters. Binding sites for NODAL signaling effectors, SMAD3/SMAD4 and FOXH1, were also conserved among human, chimpanzee, cow and dog CERI promoters. CERI orthologs were evolutionarily conserved target of WNT and NODAL signaling pathways in non-rodent mammals. Human CER1 mRNA was expressed in embryonic stem (ES) cells in the undifferentiated state and in the early endodermal lineage. CER1 upregulation in human ES cells leads to Nodal signaling inhibition associated with differentiation of human ES cells. Primate CER1 orthologs, playing a pivotal role during early
\end{abstract}

Correspondence to: Dr Masaru Katoh, Genetics and Cell Biology Section, National Cancer Center Research Institute, 5-1-1 Tsukiji, Chuo-ku, Tokyo 104-0045, Japan

E-mail: mkatoh@ncc.go.jp

Key words: bioinformatics, comparative genomics, comparative proteomics, WNT, Nodal, integrome network embryogenesis, underwent protein evolution as well as promoter evolution. These facts indicate that molecular evolution of CERI orthologs contributes to the significantly divergent scenarios of early embryogenesis in primates and rodents.

\section{Introduction}

TGFB1, TGFB2, TGFB3, NODAL, LEFTY1, LEFTY2, INHA, $I N H B A, I N H B B, I N H B C, I N H B E, A M H, B M P 2, B M P 3$, BMP4, BMP5, BMP6, BMP7, BMP8A, BMP8B, BMP10, $B M P 15, G D F 1, G D F 2$, GDF3, GDF5, GDF6, GDF7, GDF8, GDF9, GDF10, GDF11, and GDF15 are TGFß superfamily genes within the human genome (http://www.gene.ucl.ac.uk). TGFß signals are transduced through type I receptor TGFBR1 and type II receptor TGFBR2 to phosphorylate R-SMAD proteins, such as SMAD2 and SMAD3 (1-5). NODAL signals are transduced through type I receptor ACVR1B/ACVR1C and type II receptor ACVR2A/ACVR2B to phosphorylate SMAD2 or SMAD3 (6-8). BMP signals are transduced through type I receptor BMPR1A/BMPR1B/ACVR1 and type II receptor BMPR2 to phosphorylate R-SMAD proteins, such as SMAD1, SMAD5 and SMAD8 (9-11). R-SMADs, associated with SMAD4, are translocated to the nucleus to activate transcription of target genes.

CER1 (DAND4 or Cerberus 1), GREM1 (DAND2 or CKTSF1B1), GREM2 (DAND3 or CKTSF1B2), GREM3 (DAND5 or CKTSF1B3 or CER2) and NBL1 (DAND1) are secreted-type DAN domain (DAND) proteins (12-16). CER1 and GREM3 are Nodal antagonists, while GREM1 is a BMP antagonist.

TGFß superfamily signaling pathways network with WNT signaling pathway upregulating target genes based on the TCF/LEF transcriptional complex (17-28); however, WNTdependent transcriptional regulation of $D A N D$ family members remains unclear. Here, we searched for TCF/LEF-binding site within the promoter region of DAND family genes. Because triple TCF/LEF-binding sites were identified within human CERI promoter, comparative genomics analyses on CERI orthologs were further performed.

\section{Materials and methods}

WNT target gene screening. Genome sequences corresponding to human CER1, GREM1, GREM2, GREM3 and NBL1 genes 
A

\begin{tabular}{lccccc}
\hline $\begin{array}{c}\text { Human } \\
\text { gene }\end{array}$ & Alias & $\begin{array}{c}\text { Chromosomal } \\
\text { localization }\end{array}$ & RefSeq ID & $\begin{array}{c}\text { Genome } \\
\text { clone }\end{array}$ & $\begin{array}{c}\text { TCF/LEF- } \\
\text { binding sites }\end{array}$ \\
\hline CER1 & DAND4 & $9 \mathrm{p} 22.3$ & NM_005454.2 & AL390732.10 & Triple sites \\
GREM1 & DAND2 & $15 q 13.3$ & NM_013372.5 & AC090877.4 & Double sites \\
GREM2 & DAND3 & 1943 & NM_022469.3 & AL358176.22 & \\
GREM3 & DAND5 & $19 \mathrm{p} 13.13$ & NM_152654.2 & AC092069.2 & \\
NBL1 & DAND1 & $1 \mathrm{p} 36.13$ & NM_182744.1 & AL031727.43 & \\
\hline
\end{tabular}

\section{B}

TCAGTTTCTGGACGTGTTCTTTTTCGTATCCTAGGCTGATTCATGATAGTTTTGAATGATAGTATTTTTCAATTTTCCTTTGATCCCCGAAGACTCTTATTTGTGTCAATCTTTATTTAGC CTGGCCATCAATTAGTGTAAAACAAGGGGAGATGTGTGGATCAATAAGGTAAGAAACTGACTAGCCAGCGTCACACACAAAGTTATCAAACCTGGTTCTTAGAATCCACGTTCTCATGTCTGG TCF/LEF

CTCTTTGTTCAAAGGGAAAAAAAAATCAATAGGATGGCTGGAGGGAGACATTTCCCTATCCTCATTGAAGACTGATGAGCTGTCAGTCTATTACGTGGTATCCTGTTGAGGAACATGTGAACAAT TGGAAGAGTTATCTGATGACTGACCAGTTGTGAAATTAGCACCCATTAATTATTCTTAATGTGAGAGGATTAACTTGGGATTGTGAGTTTCTACGTGTGGAAAGAAAACATATTCCCTTT TCF/LEF

ACTCAGTTACTGT TTCTTCGTGTGGTGTGTGTGTGTGTGTGTGTGTGTGTGTGTGTGTGTGTGTGAATAGCCTTCCTAAAGGCCTGGGCTTTTTCTAAATGTATGCCTTTAATTTTATCCAAAAATTCTCTTACA GCAACATGTCTTGTCTATTCTATTCCAGCACAAGGCAGATGCACAGCAAATTGAGCTGACTCTAGTCCTTCTTCTGAAACAGCCATGGGAATTTAGGCAAAGATGTGTGTCTTTGCTAAT SMADs FOXH1

ACTGCTCTTTAAGCCCCAGACATAGCTAAACTCTTAGCTAATTACCCCCTGGGTCCCAGGCTTTCACTGGGGCCTTTTAAAATACACAAAACCAAAGTGACGGCAGGAGGCCATTAGCACTACAT AATTCAAGCAAACAATAAATGTGTTATTCTGCCTGGCTACTGACCACCTGCCTTCCCATCCCGCCAGGCAGGTATCTATATATACGATTTCCTTTTTCCCAGTCCTGCAGAGAATAGCCTCTC CTTTGGGCCTCATCATTTACAAAAGAAGCTTGGGCCCCTGACAGCATGCATCTCCTCTTATTTCAGCTGCTGGTACTCCTGCCTCTAGGAAAGACCACACGGCACCAGGATGGCCGCCAGAATCA

Figure 1. (A), DAND gene family. CER1, GREM1, GREM2, GREM3 and NBL1 constitute the DAND gene family. (B), Human CER1 promoter. The region corresponding to exon 1 is boxed. Three TCF/LEF-binding sites as well as SMAD- and FOXH1-binding sites are shown by over-lines. (C), Phylogenetic analyses on DAND family. Human CER1 and mouse Cer1 are significantly divergent.

were searched for with BLAST programs (http://www.ncbi. nlm.nih.gov) as described previously (29-33). TCF/LEFbinding sites within the 5'-flanking promoter region of the above genes were searched for based on bioinformatics and manual inspection as described previously (34-38).

Identification of chimpanzee CER1 ortholog. Chimpanzee genome sequences homologous to human $C E R I$ were searched for with BLAST programs as described previously (39-42). Exon-intron boundaries were determined based on the consensus sequence of exon-intron junctions ('gt ... ag' rule of intronic sequence) and codon usage within the coding region as described previously (43-46). Coding sequence of chimpanzee CER1 was determined by assembling exonic regions.

Comparative proteomics analysis. Phylogenetic analyses on mammalian DAND family members were performed by using the CLUSTALW program.

Comparative genomics analyses. Promoter region of mammalian CER1 orthologs were aligned by using the Genetyx program and manual curation. TCF/LEF-binding sites within the promoter region were determined as mentioned above.

In silico expression analysis. Expressed sequence tags (ESTs) derived from human $C E R I$ genes were searched for by using the BLAST programs. The sources of CERI ESTs were listed up for in silico expression analysis.

\section{Results}

Screening of the TCF/LEF-binding site within promoter region of DAND family genes. Human CER1 RefSeq (NM_005454.2),
GREM1 RefSeq (NM_013372.5), GREM2 RefSeq (NM_022469.3), GREM3 RefSeq (NM_152654.2) and NBL1 RefSeq (NM_182744.1) were used as query sequences for the BLAST programs to identify genome clones corresponding to $D A N D$ family genes. The 5'-flanking promoter region of human CER1, GREM1, GREM2, GREM3 and NBL1 genes were identified within AL390732.10, AC090877.4, AL358176.22, AC092069.2 and AL031727.43 genome sequences, respectively (Fig. 1A). TCF/LEF-binding sites within the 5'-promoter region of human CER1, GREM1, GREM2, GREM3 and NBL1 genes were then searched for based on manual inspection as described previously (34-38). Triple TCF/LEF-binding sites were identified within human CERl promoter (Fig. 1B).

Identification of the chimpanzee CERI gene. BLAST programs using human CER1 RefSeq revealed that chimpanzee CERI gene was located within NW_111298.1 genome sequence. Exon-intron boundaries of chimpanzee CERl gene were determined based on the consensus sequence of exonintron junctions. Exon 1 corresponded to nucleotide position 289994-289443 of NW_111298.1 genome sequence, while exon 2 corresponded to nucleotide position 287557-286907. Chimpanzee CER1 gene was found consisting of two exons.

LOC473172 predicted sequence (XM_528542.1), corresponding to 5 '-flanking regions, intron 1 and exon 2 of chimpanzee CERl gene, was not the correct chimpanzee CER1 sequence. Complete coding sequence (CDS) of chimpanzee CER1 was determined by assembling nucleotide sequences of two exons in this study (Fig. 2A).

Genetyx program revealed that nucleotide position 46-849 was the coding region of chimpanzee CER1 complete CDS (Fig. 2A). Chimpanzee CERl gene was found to encode a 267-amino-acid protein. 
A

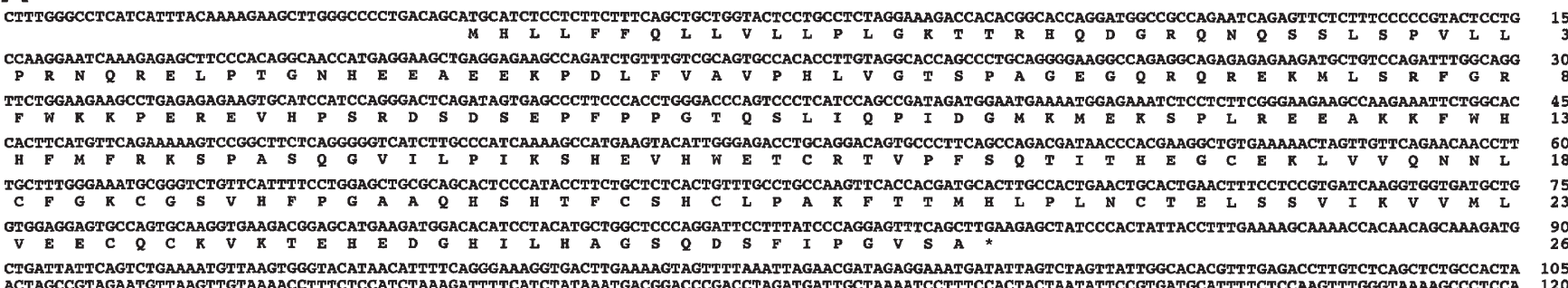

CTGATTATTCAGTCTGAAAATGTTAAGTGGGTACATAACATTTTCAGGGAAGGTGACTTGAAAAGTAGTTTTAAATTAGAACGATAGAGGAAATGATATTAGTCTAGTTATTGGCACACGTTTGAGACCTTGTCTCAGCTCTGCCACTA 1050

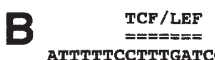

ATTTTTCCTTTGATCCCCGAAGACTCTTATTTGTGTCAATCTTTATTTAGCCTGGCCATCAATTAGTGTAAAACAAGGGGAGATGATGTGGATCAATAAGGTAAGAACTGACTAGCCAGCGTCACACCCAAAGTTATCAAACCTGGTT TCF $/$ LEF

CTTAGAATCCGCGTTCTCATGTCTGGCTCTTTGTTTAAAGGGAAAAAAAAATCAATAGGATGGCTGGAGGGAGACATTTCCCTATCCTCATTGAAGACTGATGAGCTATCAGTCTATTACGTGGTATCCTGTTGAGAAACGTGAACAATT GGAAGAGTTTATCTGATGACTGACCAGTTGTGAAATTTAACACCCATTAATTGATTCTTAATGTGAGAGGATTAACTTGGGAATTGTGAGTTTCTATGTGTGGAAAGGAAAACATATTCCCTTTACTCAGTTACCATGCAAAAATCAATA $\underset{T C E / L E F}{T=m=m=m}$

ACCCCAGTTCGCATGTAAGTAAGCAACAATATGTCCCTTGCTCAAAAAAATTAACATCCTTCTAGCTCTTCCTITCTTCTCCTTACAACTITGATATACTTCTTCGTGTGTCTGTGTGTGTGTGTGTGTGTGTGTGTGTGTGTGTGTGTG TGAATAGCCTTCCTAAAAGCCTGGGCTTTTTCTAAATGTATGCCTTTAATTTTTATCCAATATACTTCTCTTACAGCAACATGTCTTGTCTATTCTATTCCAGCACAAGGCAGATGCGCAGCAAATGTGAGCTGACTCTAGTCCTTCTTC SMADs FOXH1

TGAAAACAGCCATGGGAATTTIAGGCAAAGAATGTGTTGTCTTTGCTAATACTACTCTITAAGCCCCAGACATAGCTAAACTCTTAGCTAATTACCCCCTGGGTCCCAGGCTTTCACTGGGCCTTTTAAAATTACACAAAACCAAAGTGA CGGCAGGAGGCCATTAGCACTACATAATTCAAGCAAACAATIAAATGTGTTTATTCTGCCTGGCTACTGACCACCTGCCTTCCCATCCCGCCAGGCAGGTATCTATATATACGATTTCCTTTITCCCAGTCCTGCAGAGAATGAGCCTCTC CTTTGGGCCTCATCATTTACAAAAGAAGCTTGGGCCCCTGACAGCATGCATCTCCTCTTCTTTCAGCTGCTGGTACTCCTGCCTCTAGGAAAGACCACACGGCACCAGGATGGCCGCCAGAATCAGAGTTCTCTTTCCCCCGTACTCCTG

Figure 2. (A), Nucleotide and amino-acid sequences of chimpanzee CER1. Nucleotides and amino-acid residues are numbered on the right. (B), Chimpanzee CER1 promoter. The region corresponding to exon 1 is boxed. Three TCF/LEF-binding sites as well as SMAD- and FOXH1-binding sites are shown by over-lines.
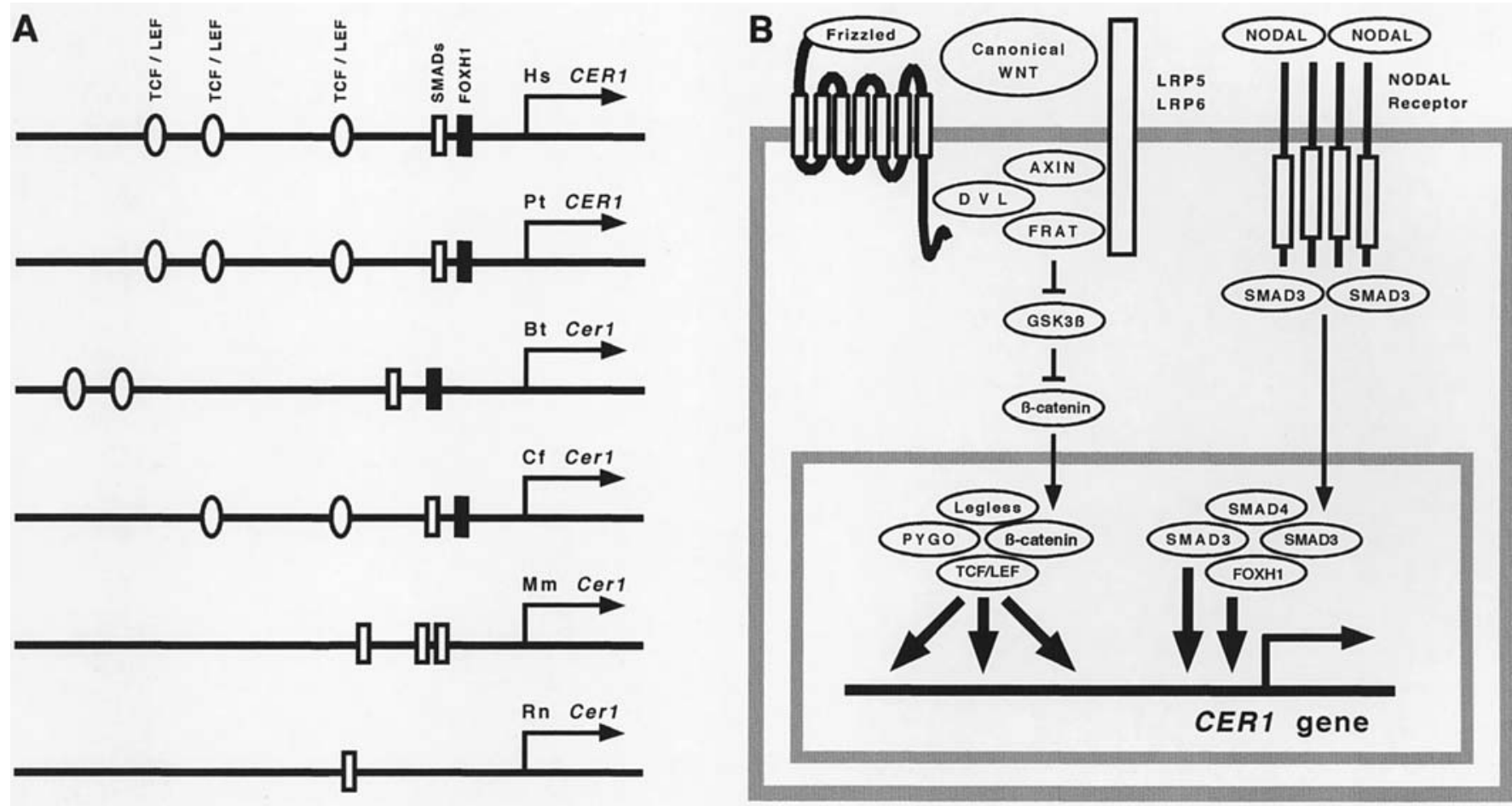

Figure 3. (A), Schematic representation of 5'-promoter region of mammalian CERl orthologs. Hs, human; Pt, chimpanzee; Bt, cow; Cf, dog; Mm, mouse; Rn, rat. TCF/LEF-binding site (oval), SMAD-binding site (open box) and FOXH1-binding site (closed box) are shown. Human, chimpanzee, cow and dog CER1 orthologs are evolutionarily conserved target of WNT and NODAL signaling pathways. (B), Schematic representation of WNT and NODAL signaling pathways in human ES cells. WNT and NODAL signaling pathways are necessary for the maintenance of human ES cells, and CER1 is the common target of WNT and NODAL signaling pathways.

Comparative proteomics analysis on mammalian DAND family members. Phylogenetic analysis revealed that GREM1, GREM2 and NBL1 orthologs were more related to each other than the CER1 and GREM3 orthologs (Fig. 1C). Chimpanzee CER1 showed $97.8 \%$ and $68.5 \%$ total-aminoacid identity with human CER1 and mouse Cer1, respectively. These facts indicate that primate CER 1 orthologs were significantly divergent from rodent Cer1 orthologs.
Expression of human CERI mRNA. In silico expression analyses were performed to investigate expression of human CER1. Human CERI mRNA was expressed in embryonic stem (ES) cells in the undifferentiated state and in the early endodermal lineage.

Comparative genomics analyses on CER1 promoters. Human $C E R 1$ promoter and chimpanzee $C E R I$ promoter were located 
within AL390732.10 and NW_111298.1 genome sequences, respectively, as mentioned above. BLAST programs revealed that the cow Cerl, dog Cerl, mouse Cerl and rat Cerl promoters were located within AC173174.3, NW_876253.1, AL670958.4 and AC091341.6 genome sequences, respectively. Phylogenetic analysis on the 5'-promoter region of mammalian $C E R I$ orthologs revealed that human, chimpanzee, cow and dog CERI promoters were significantly divergent from mouse and rat Cerl promoters.

GC content of human CERI promoter was $42.8 \%$, that of chimpanzee CER1 promoter was $42.9 \%$, that of cow Cer 1 promoter was $45.5 \%$, that of dog Cerl promoter was $41.2 \%$, that of mouse Cerl promoter was $47.0 \%$, and that of rat Cerl promoter were $47.7 \%$. GC content of human, chimpanzee, cow and $\operatorname{dog} C E R I$ promoters were lower than those of mouse and rat Cerl promoters.

Three TCF/LEF-binding sites within human CERl promoter were conserved in chimpanzee CER1 promoter, two in cow and dog Cerl promoters, but not in rodent Cerl promoters (Fig. 3A).

Because WNT and NODAL signaling pathways play a key role in the maintenance of human ES cells (47), we next investigated the binding sites for NODAL signaling effectors, SMAD3/SMAD4 and FOXH1. SMAD3/SMAD4-binding site was conserved among mammalian CERI promoters. On the other hand, FOXH1-binding site was conserved only among human, chimpanzee, cow and dog CERl promoters, but not in mouse and rat Cerl promoters (Fig. 3A).

These facts indicate that CERI orthologs were evolutionarily conserved target of WNT and NODAL signaling pathways in human, chimpanzee, cow and dog.

\section{Discussion}

TCF/LEF-binding site within the promoter region of $D A N D$ family genes, including CER1, GREM1, GREM2, GREM3 and $N B L 1$, were searched for by using bioinformatics and human intelligence in this study. Because triple TCF/LEFbinding sites were identified within human CERl promoter (Fig. 1B), comparative genomics analyses on CERI orthologs were further performed.

Chimpanzee CERl gene, consisting of two exons, was identified within NW_111298.1 genome sequence. Because XM_528542.1 was not a correct coding sequence for chimpanzee CER1, complete CDS of chimpanzee CER1 was determined by assembling exonic regions (Fig. 2A). Chimpanzee CER1 gene was found to encode a 267 -aminoacid protein showing $97.8 \%$ and $68.5 \%$ total-amino-acid identity with human CER1 and mouse Cer1, respectively. Phylogenetic analysis on human and mouse DAND family members next revealed that human CER1 and mouse Cer1 were significantly divergent (Fig. 1C). These facts clearly indicate that the CER1 protein evolution has occurred during mammalian evolution.

Three TCF/LEF-binding sites within human CERI promoter were conserved in chimpanzee CERI promoter, two sites in cow and dog Cerl promoters, but no site in rodent Cerl promoters (Fig. 3A). Binding sites for NODAL signaling effectors, SMAD3/SMAD4 and FOXH1, were also conserved among human, chimpanzee, cow and $\operatorname{dog} C E R I$ promoters
(Fig. 3A). Based on these facts, non-rodent mammalian CERl orthologs were identified as the evolutionarily conserved target of WNT and NODAL signaling pathways.

CER1 mRNA was expressed in human ES cells in the undifferentiated state and in the early endodermal lineage as mentioned in the Results. WNT and NODAL signaling pathways are indispensable for human ES cells (47), and CER1 is the common target of WNT and NODAL signaling pathways (Fig. 3B). Because CER1 upregulation in human ES cells leads to Nodal signaling inhibition associated with endodermal differentiation, CER1 is a key molecule for the maintenance of human ES cells. CER1 is the pharmacogenomics target in the field of regenerative medicine.

Primate CER1 orthologs, playing a pivotal role during early embryogenesis, underwent protein evolution as well as promoter evolution. These facts indicate that molecular evolution of CERI orthologs contributes to the significantly divergent scenarios of early embryogenesis in primates and rodents.

\section{References}

1. Heldin $\mathrm{CH}$, Miyazono $\mathrm{K}$ and ten Dijke P: TGFß signaling from cell membrane to nucleus through SMAD proteins. Nature 390: 465-471, 1997.

2. Massague J: TGFß signal transduction. Annu Rev Biochem 67: 753-791, 1998.

3. Massague J: How cells read TGFß signals. Nat Rev Mol Cell Biol 1: 169-178, 2000.

4. Moustakas A, Souchelnytskyi S and Heldin $\mathrm{CH}$ : Smad regulation in TGFß signal transduction. J Cell Sci 114: 4359-4369, 2001.

5. Miyazono K, ten Dijke P and Heldin $\mathrm{CH}$ : Divergence and convergence of TGFß/BMP signaling. J Cell Physiol 187: 265-276, 2001.

6. Whiteman M: Nodal signaling in early vertebrate embryos: themes and variations. Dev Cell 1: 605-617, 2001.

7. Reissmann E, Jornvall H, Blokzijl A, et al: The orphan receptor ALK7 and Activin receptor ALK4 mediate signaling by Nodal proteins during vertebrate development. Genes Dev 15: 2010-2022, 2001

8. Norris DP, Brennan J, Bikoff EK and Robertson EJ: The Foxh1dependent autoregulatory enhancer controls the level of Nodal signaling in the mouse embryo. Development 129: 3455-3468, 2002.

9. Zhang J and Li L: BMP signaling and stem cell regulation. Dev Biol 284: 1-11, 2005.

10. Varga AC and Wrana JL: The disparate role of BMP in stem cell biology. Oncogene 24: 5713-5721, 2005.

11. Katoh $\mathrm{Y}$ and Katoh $\mathrm{M}$ : Comparative genomics on $B M P 4$ orthologs. Int J Oncol 27: 581-585, 2005.

12. Lah M, Brodnicki T, Maccarone P, et al: Human cerberus related gene CER1 maps to chromosome 9. Genomics 55: 364-366, 1999.

13. Topol LZ, Modi WS, Koochekpour S and Blair DG: DRM/ GREMLIN (CKTSF1B1) maps to human chromosome 15 and is highly expressed in adult and fetal brain. Cytogenet Cell Genet 89: 79-84, 2000

14. Katoh $\mathrm{M}$ and Katoh M: Identification and characterization of human CKTSF1B2 and CKTSF1B3 genes in silico. Oncol Rep 12: 423-427, 2004.

15. Marques S, Borges AC, Silva AC, et al: The activity of the Nodal antagonist Cerl-2 in the mouse node is required for correct L/R body axis. Genes Dev 18: 2342-2347, 2004.

16. Katoh M and Katoh M: Comparative genomics on Norrie disease gene. Int J Mol Med 15: 885-889, 2005.

17. Katoh M: $W N T$ and $F G F$ gene clusters. Int J Oncol 21: 1269-1273, 2002.

18. Katoh M: Regulation of WNT signaling molecules by retinoic acid during neuronal differentiation in NT2 cells: threshold model of WNT action. Int J Mol Med 10: 683-687, 2002.

19. Heller RS, Klein T, Ling Z, Heimberg H, Katoh M, Madsen OD and Serup P: Expression of WNT, Frizzled, sFRP, and DKK genes in adult human pancreas. Gene Expr 11: 141-147, 2003. 
20. Katoh M: WNT2 and human gastrointestinal cancer. Int J Mol Med 12: 811-816, 2003.

21. Katoh $\mathrm{M}$ and Katoh M: Identification and characterization of human BCL9L gene and mouse Bcl9l gene in silico. Int J Mol Med 12: 643-649, 2003.

22. Garciadiego-Cazares D, Rosales C, Katoh M and ChimalMonroy J: Coordination of chondrocyte differentiation and joint formation by $\alpha 5 \beta 1$ integrin in the developing appendicular skeleton. Development 131: 4735-4742, 2004.

23. Katoh $\mathrm{M}$ and Katoh M: Comparative genomics on WNT8A and WNT8B genes. Int J Oncol 26: 1129-1133, 2005.

24. Katoh M: Molecular evolution of WNT2B orthologs. Int J Oncol 26: 1135-1139, 2005.

25. Katoh M: Comparative genomics on WNT3-WNT9B gene cluster. Int J Mol Med 15: 743-747, 2005.

26. Swain RK, Katoh M, Medina A and Steinbeisser H: Xenopus frizzled-4S, a splicing variant of Xfz4, is a context-dependent activator and inhibitor of Wnt/B-catenin signaling. Cell Commun Signal 3: 12, 2005.

27. Katoh M: Epithelial-mesenchymal transition in gastric cancer. Int J Oncol 27: 1677-1683, 2005.

28. Katoh M: WNT2B: comparative integromics and clinical application. Int J Mol Med 16: 1103-1108, 2005.

29. Katoh M: Paradigm shift in gene-finding method: from benchtop approach to desk-top approach. Int J Mol Med 10: 677-682, 2002.

30. Katoh $\mathrm{M}$ and Katoh M: Evolutionary conservation of $C C N D 1$ ORAOV1-FGF19-FGF4 locus from zebrafish to human. Int J Mol Med 12: 45-50, 2003.

31. Katoh M and Katoh M: $C L D N 23$ gene, frequently down-regulated in intestinal-type gastric cancer, is a novel member of CLAUDIN gene family. Int J Mol Med 11: 683-689, 2003.

32. Katoh $\mathrm{M}$ and Katoh $\mathrm{M}$ : Identification and characterization of human MPP7 gene and mouse Mpp7 gene in silico. Int $\mathrm{J}$ Mol Med 13: 333-338, 2004.
33. Katoh $\mathrm{M}$ and Katoh M: Identification and characterization of Crumbs homolog 2 gene at human chromosome 9q33.3. Int J Oncol 24: 743-749, 2004

34. Katoh Y and Katoh M: Comparative genomics on DKK1 orthologs. Int J Oncol 27: 275-279, 2005.

35. Katoh $\mathrm{Y}$ and Katoh M: Comparative genomics on DKK2 and DKK4 orthologs. Int J Mol Med 16: 477-481, 2005.

36. Katoh $\mathrm{M}$ and Katoh M: Comparative genomics on FGF20 orthologs. Oncol Rep 14: 287-290, 2005.

37. Katoh M and Katoh M: Comparative genomics on $F G F 8$, FGF17, and FGF18 orthologs. Int J Mol Med 16: 493-496, 2005.

38. Katoh Y and Katoh M: Comparative genomics on FGF16 orthologs. Int J Mol Med 16: 959-963, 2005.

39. Katoh $\mathrm{M}$ and Katoh $\mathrm{M}$ : Identification and characterization of human HES2, HES3, and HES5 genes in silico. Int J Oncol 25: 529-534, 2004.

40. Katoh M and Katoh M: Identification and characterization of human HESL, rat Hesl and rainbow trout hesl genes in silico. Int J Mol Med 14: 747-751, 2004.

41. Katoh $\mathrm{M}$ and Katoh M: Comparative genomics on WNT5A and WNT5B genes. Int J Mol Med 15: 749-753, 2005.

42. Katoh Y and Katoh M: Comparative genomics on WNT11 gene. Int J Mol Med 15: 879-883, 2005.

43. Katoh Y and Katoh M: Comparative genomics on VANGL1 and VANGL2 genes. Int J Oncol 26: 1435-1440, 2005.

44. Katoh Y and Katoh M: Comparative genomics on SFRP1 orthologs. Int J Oncol 27: 861-865, 2005.

45. Katoh Y and Katoh M: WNT antagonist, SFRP1, is Hedgehog signaling target. Int J Mol Med 17: 171-175, 2006.

46. Katoh Y and Katoh M: Comparative genomics on HHIP family orthologs. Int J Mol Med 17: 391-395, 2006.

47. James D, Levine AJ, Besser D and Hemmati-Brivanlou A: TGFß/ activin/nodal signaling is necessary for the maintenance of pluripotency in human embryonic stem cells. Development 132: 1273-1282, 2005. 